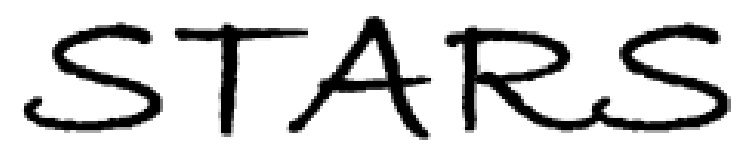

University of Central Florida

STARS

$1-1-2014$

\title{
Time-dependent density-functional theory of exciton-exciton correlations in the nonlinear optical response
}

Volodymyr Turkowski

University of Central Florida

Michael N. Leuenberger

University of Central Florida

Find similar works at: https://stars.library.ucf.edu/facultybib2010 University of Central Florida Libraries http://library.ucf.edu

This Article is brought to you for free and open access by the Faculty Bibliography at STARS. It has been accepted for inclusion in Faculty Bibliography 2010 s by an authorized administrator of STARS. For more information, please contact STARS@ucf.edu.

\section{Recommended Citation}

Turkowski, Volodymyr and Leuenberger, Michael N., "Time-dependent density-functional theory of excitonexciton correlations in the nonlinear optical response" (2014). Faculty Bibliography 2010s. 6198.

https://stars.library.ucf.edu/facultybib2010/6198

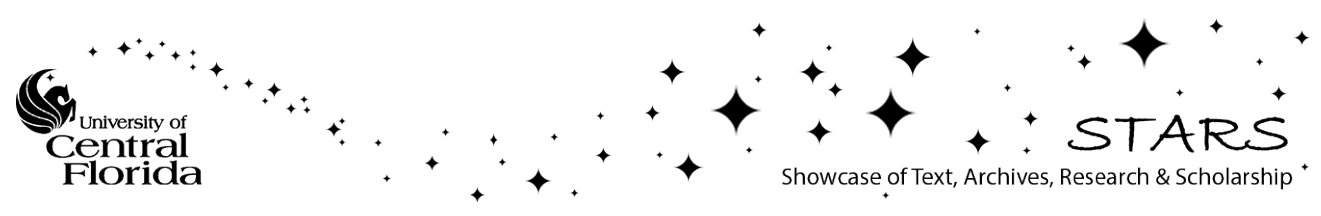




\title{
Time-dependent density-functional theory of exciton-exciton correlations in the nonlinear optical response
}

\author{
Volodymyr Turkowski and Michael N. Leuenberger* \\ Department of Physics and NanoScience and Technology Center, University of Central Florida, Orlando, Florida 32816, USA
}

(Received 15 June 2013; revised manuscript received 19 December 2013; published 19 February 2014)

\begin{abstract}
We analyze possible nonlinear exciton-exciton correlation effects in the optical response of semiconductors by using a time-dependent density-functional theory (TDDFT) approach. For this purpose we derive the nonlinear (third-order) TDDFT equation for the excitonic polarization. In this equation the nonlinear time-dependent effects are described by the time-dependent (nonadiabatic) part of the effective exciton-exciton interaction, which depends on the exchange-correlation (XC) kernel. We apply the approach to study the nonlinear optical response of a GaAs quantum well. In particular, we calculate the two-dimensional Fourier spectrum (2DFS) of the system and compare it with experimental data. We find that the memory effects play a crucial role in this response, and in particular that it is necessary to use a nonadiabatic XC kernel to describe excitonic bound states, biexcitons, which are formed due to the retarded TDDFT exciton-exciton interaction.
\end{abstract}

DOI: 10.1103/PhysRevB.89.075309

PACS number(s): 71.15.Mb, 71.35.-y, 73.21.-b

\section{INTRODUCTION}

The nonlinear optical response of semiconductors is an important problem of modern condensed matter physics, in particular due to the necessity to describe correctly the ultrafast laser pulse experiments [1], including the four-wave mixing (FWM) case [2], in nanostructures and other systems. This problem is a part of a more general problem of the nonequilibrium nonlinear behavior of quantum matter at ultrashort time scales, one of the most complicated and important problems of modern physics. It is extremely important to understand this behavior from both fundamental science and technological points of view, and the FWM experiment is one of the most powerful and promising tools currently used for this purpose. A fundamental process in the FWM experiments is generation of multiple excitonic states, which can have a variety of practical applications from optoelectronic devices [3] to quantum computing [4]. In many cases, including the ultrafast response, the nonlinear effects, which come from the time-dependent exciton-exciton interaction, are very important. To our knowledge, these were studied only in the framework of many-body effective models by deriving and solving the effective thirdorder equation for the polarization (see, e.g., Refs. $[2,5,6]$ ). In particular, it was shown that the nonlinear effects come from the time-dependent exciton-exciton interactions defined by the corresponding correlation functions. Due to the complexity of the problem, the time-dependent correlation function is usually approximated by a phenomenological expression. Since the optical response strongly depends on the form of this expression [6], it is highly desirable to obtain the form of the effective interaction from a simple but fundamental approach that does not include (or at least includes a minimal number of) phenomenological parameters. For this purpose, we propose to use TDDFT [7]. It was already successfully applied to study the excitonic effects in the frameworks of the time-dependent optimized effective potential approach [8], in combination with the Bethe-Salpeter equation [1], and by solving the TDDFT version of the semiconductor Bloch equations (SBEs,

*Corresponding author: mleuenbe@mail.ucf.edu see, e.g., Ref. [9]) [10,11]. We recently generalized the last approach to the biexcitonic case [12]. In most of these papers, the contribution of the exciton-exciton interaction to the optical absorption spectrum was neglected. Only in Ref. [10] the nonlinear excitonic effects were taken into account by solving a nonlinear system of the TDDFT-SBEs. However, it was difficult to identify the nonlinear contribution from such a general solution. In addition, since the adiabatic approximation was used, the time-resolved exciton-exciton interaction was neglected in this case. As we show below, the nonadiabaticity of the $\mathrm{XC}$ kernels is a crucial requirement for the development of a theory based on TDDFT to describe nonlinear correlation effects including the formation of exciton-exciton bound states (biexcitons). Another requirement, which follows from all the studies mentioned above, is the necessity to go beyond the LDA and GGA approximation in order to obtain even single-exciton effects.

Currently, possible structures of the nonadiabatic XC potentials are much less understood compared to the cases of static DFT and adiabatic TDDFT. However, the frequency dependence of the XC potential is not only important for the description of the exciton-exciton interaction but also for various static problems such as multiple excitations [12-16] and time-dependent problems when the system response is analyzed (see, e.g., Ref. [17]). In this work we use the available exact knowledge of the asymptotic limits of the XC kernels at low and high frequencies and some experimental data to construct the nonadiabatic part of the kernels that are able to describe the effects of the exciton-exciton correlations within TDDFT, including biexcitonic bound states. We test the approach by calculating the response of a GaAs quantum well in a FWM experiment and demonstrate that the approach is capable of describing the main features of the spectrum. The method proposed here can be used in a more general case to study the nonadiabatic response within TDDFT.

\section{METHOD}

In order to derive the nonlinear equation for the polarization, we use the density-matrix representation of TDDFT. For 
simplicity we consider the three-band case (two heavy- and light-hole valence bands and one conduction band), though the generalization to more bands is straightforward. In the density-matrix TDDFT, one looks for the solution of the time-dependent Kohn-Sham (KS) equation

$$
i \partial \Psi(\mathbf{r}, t) / \partial t=\hat{H}(\mathbf{r}, t) \Psi(\mathbf{r}, t)
$$

as a linear combination of the wave functions which corresponds to the static solution,

$$
E_{l} \psi^{l(0)}(\mathbf{r})=\hat{H}(\mathbf{r}, t=0) \psi^{l(0)}(\mathbf{r}, t),
$$

where $l$ is the band number. In the last equation,

$$
\hat{H}(\mathbf{r}, t)=-\frac{\nabla^{2}}{2 m}+V(\mathbf{r}, t)+V_{\mathrm{H}}[n](\mathbf{r}, t)+V_{\mathrm{XC}}[n](\mathbf{r}, t)
$$

is the time-dependent KS Hamiltonian, where we set $\hbar=1$,

$$
V(\mathbf{r}, t)=V_{\text {nucl }}(\mathbf{r})+V_{\text {ext }}(\mathbf{r}, t)
$$

is the sum of the static atomic potential and the timedependent external potential, and $V_{\mathrm{H}}(\mathbf{r}, t)$ and $V_{\mathrm{XC}}(\mathbf{r}, t)$ are the time-dependent Hartree and XC potentials, which depend on the single-particle density $n(\mathbf{r}, t)$ (which should be found self-consistently from the $\mathrm{KS}$ wave functions). We use the dipole approximation for the external perturbation arising from the electric field pulse,

$$
V_{\mathrm{ext}}(\mathbf{r}, t)=-\mathbf{r} E(t),
$$

which corresponds to the case of high frequencies, comparing to the spacing between the energy levels. In the three-band case, the wave function can be approximated as a linear combination of the valence and the conduction electron wave functions:

$$
\Psi_{\mathbf{k}}(\mathbf{r}, t)=\sum_{l=v_{1}, v_{2}, c} c_{\mathbf{k}}^{l}(t) \psi_{\mathbf{k}}^{l(0)}(\mathbf{r}),
$$

and the problem reduces to the solution of the Liouville equation

$$
i \frac{\partial \rho_{\mathbf{k}}^{l m}}{\partial t}=[H, \rho]_{\mathbf{k}}^{l m},
$$

where

$$
H_{\mathbf{k}}^{l m}(t)=\int d \mathbf{r} \psi_{\mathbf{k}}^{l(0) *}(\mathbf{r}) \hat{H}(\mathbf{r}, t) \psi_{\mathbf{k}}^{m(0)}(\mathbf{r})
$$

are the matrix elements of the Hamiltonian with respect to the static wave functions and

$$
\rho_{\mathbf{k}}^{l m}(t)=c_{\mathbf{k}}^{l}(t) c_{\mathbf{k}}^{m *}(t)
$$

is the $3 \times 3$ density matrix, whose diagonal elements correspond to the band occupancies

$$
\begin{gathered}
n_{v_{i}}(t)=\rho_{\mathbf{k}}^{v_{i} v_{i}}(t) \quad(i=l, h), \\
n_{c}(t)=\rho_{\mathbf{k}}^{c c}(t)=1-\rho_{\mathbf{k}}^{v_{1} v_{1}}(t)-\rho_{\mathbf{k}}^{v_{2} v_{2}}(t),
\end{gathered}
$$

and the nondiagonal elements-to the direct and the inverse (including excitonic) coherences $\rho_{\mathbf{k}}^{c v}(t)$ and $\rho_{\mathbf{k}}^{v c}(t)=$ $\rho_{\mathbf{k}}^{c v *}(t)$. For simplicity, we take into account only the vertical transitions since in most cases the photon momentum can be neglected. The system of independent Liouville equations have the form of the SBEs [9] for the excited electron density $n_{c}(t)$ and polarizations $\rho_{\mathbf{k}}^{c v}(t)$ (for details, see Ref. [8]). We shall consider the nonlinear equation for the exciton polarization by taking into account the terms up to the third order in $\rho_{\mathbf{k}}^{c v}(t)$ [or, more precisely, in $c_{\mathbf{k}}^{c}(t)$ ]. This can be done by expanding the nonlinear part $V_{\mathrm{XCk}}^{l m}(t)$ of the matrix elements $H_{\mathbf{k}}^{l m}(t)$ in powers of the fluctuating particle density

$$
\delta n(\mathbf{r}, t)=n(\mathbf{r}, t)-n(\mathbf{r}, t=0),
$$

i.e.,

$$
V_{\mathrm{XCk}}^{l m}(t)=\int d \mathbf{r} \psi_{\mathbf{k}}^{l(0) *}(\mathbf{r})\left(\sum_{n} \frac{1}{n !} \frac{\delta^{n} V_{\mathrm{XC}}}{\delta n^{n}} \delta n^{n}\right) \psi_{\mathbf{k}}^{m(0)}(\mathbf{r}),
$$

where the expression in the brackets is the Taylor expansion of the functional $V_{\mathrm{XC}}$, including the space-time integration over the internal variables. As it follows from the definition of the wave function and the density matrix, one can use the expansion of the wave function in terms of the $c$ coefficients in Eq. (6) and express the density fluctuation in terms of the elements of the density matrix:

$$
\begin{aligned}
\delta n(\mathbf{r}, t)= & \sum_{k<k_{F}}\left[\left|\Psi_{\mathbf{k}}(\mathbf{r}, t)\right|^{2}-\left|\Psi_{\mathbf{k}}(\mathbf{r}, t=0)\right|^{2}\right] \\
\simeq & \sum_{k<k_{F}}\left[\rho_{\mathbf{k}}^{c v_{1}}(t) \psi_{\mathbf{k}}^{c(0)}(\mathbf{r}) \psi_{\mathbf{k}}^{v_{1}(0) *}(\mathbf{r})\right. \\
& \left.+\rho_{\mathbf{k}}^{c v_{2}}(t) \psi_{\mathbf{k}}^{c(0)}(\mathbf{r}) \psi_{\mathbf{k}}^{v_{2}(0) *}(\mathbf{r})\right] .
\end{aligned}
$$

In the last expression we neglect the complex conjugated terms, since they correspond to the exciton de-excitations and are small comparing to the presented terms when the photon frequency is close to the (usually large) energy gap [10,11]. Next, taking into account the fact that the exciton (polarization) functions are related to the density matrix elements as $P_{1 \mathrm{k}}(t)=$ $\rho_{\mathbf{k}}^{c v_{1}}(t), P_{2 \mathbf{k}}(t)=\rho_{\mathbf{k}}^{c v_{2}}(t)$, Eq. (7) for the matrix elements $\rho_{\mathbf{k}}^{c v_{1}}(t)$ and $\rho_{\mathbf{k}}^{c v_{2}}(t)$ in the third-order approximation [see Eqs. (13) and (14)] correspond to the following equations for the polarization:

$$
\begin{aligned}
i \frac{\partial}{\partial t} P_{1 \mathbf{k}}(t)= & {\left[\varepsilon_{\mathbf{k}}^{c}-\varepsilon_{\mathbf{k}}^{v_{1}}\right] P_{1 \mathbf{k}}(t) } \\
& +\sum_{\mathbf{q}} \int d t^{\prime} \alpha_{11 \mathbf{k q}}\left(t, t^{\prime}\right) P_{1 \mathbf{q}}\left(t^{\prime}\right)+\mathbf{d}_{\mathbf{k}}^{c v 1} \mathbf{E}(t) \\
& +\sum_{\mathbf{q}, \mathbf{p}, \mathbf{Q}} P_{1 q}^{*}(t) \int d t^{\prime} F_{k q p Q}^{11}\left(t, t^{\prime}\right) P_{1 p}\left(t^{\prime}\right) P_{1 Q}\left(t^{\prime}\right) \\
& +\sum_{\mathbf{q}, \mathbf{p}, \mathbf{Q}} P_{2 q}^{*}(t) \int d t^{\prime} F_{k q p Q}^{12}\left(t, t^{\prime}\right) P_{1 p}\left(t^{\prime}\right) P_{2 Q}\left(t^{\prime}\right),
\end{aligned}
$$

and similar equation for the polarization $P_{2 \mathbf{k}}(t)$ with interchange of 1 and 2 . In these equations,

$$
\alpha_{11 \mathbf{k q}}\left(t, t^{\prime}\right)=2 \int d \mathbf{r} \int d \mathbf{r}^{\prime} \varphi_{1 \mathbf{k}}^{*}(\mathbf{r}) f_{\mathrm{XC}}\left(\mathbf{r}, t ; \mathbf{r}^{\prime}, t^{\prime}\right) \varphi_{1 \mathbf{k}}\left(\mathbf{r}^{\prime}\right)
$$


and

$$
\begin{aligned}
F_{11 \mathbf{k q}}\left(t, t^{\prime}\right)= & \frac{1}{3 !} \int d \mathbf{r} \int d \mathbf{r}^{\prime} \int d \mathbf{r}^{\prime \prime} \int d \mathbf{r}^{\prime \prime \prime} \varphi_{1 \mathbf{k}}^{*}(\mathbf{r}) \varphi_{1 \mathbf{q}}^{*}\left(\mathbf{r}^{\prime}\right) \\
& \times f_{\mathrm{XC}}^{\prime \prime}\left(\mathbf{r}, t ; \mathbf{r}^{\prime}, t ; \mathbf{r}^{\prime \prime}, t^{\prime} ; \mathbf{r}^{\prime \prime \prime}, t^{\prime}\right) \varphi_{1 \mathbf{p}}\left(\mathbf{r}^{\prime \prime}\right) \varphi_{\mathbf{Q}}\left(\mathbf{r}^{\prime \prime \prime}\right), \\
F_{12 \mathbf{k q}}\left(t, t^{\prime}\right)= & \frac{1}{3 !} \int d \mathbf{r} \int d \mathbf{r}^{\prime} \int d \mathbf{r}^{\prime \prime} \int d \mathbf{r}^{\prime \prime \prime} \varphi_{1 \mathbf{k}}^{*}(\mathbf{r}) \varphi_{2 \mathbf{q}}^{*}\left(\mathbf{r}^{\prime}\right) \\
& \times f_{\mathrm{XC}}^{\prime \prime}\left(\mathbf{r}, t ; \mathbf{r}^{\prime}, t ; \mathbf{r}^{\prime \prime}, t^{\prime} ; \mathbf{r}^{\prime \prime \prime}, t^{\prime}\right) \\
& \times\left[\varphi_{1 \mathbf{p}}\left(\mathbf{r}^{\prime \prime}\right) \varphi_{\mathbf{Q}}\left(\mathbf{r}^{\prime \prime \prime}\right)+\varphi_{1 \mathbf{Q}}\left(\mathbf{r}^{\prime \prime}\right) \varphi_{\mathbf{p}}\left(\mathbf{r}^{\prime \prime \prime}\right)\right]
\end{aligned}
$$

are the corresponding TDDFT electron-hole and twoelectron/two-hole scattering (interaction) potentials. In the last equations, $\varphi_{l \mathbf{k}}(\mathbf{r})=\psi_{k}^{c(0)}(\mathbf{r}) \psi_{k}^{v_{1}(0)}(\mathbf{r})$ are the Kohn-Sham "exciton" wave functions, and $f_{\mathrm{XC}}^{\prime \prime}$ is the second derivative of the XC kernel with respect to the charge density. Similar equations can be written for the polarization $P_{2 \mathbf{k}}(t)$ with interchange of 1 and 2 in Eqs. (15)-(18). Let us briefly discuss the meaning of the approximation which results in Eq. (15). This equation follows from the following nonequilibrium part of the XC energy:

$$
\begin{aligned}
\delta E_{\mathrm{XC}}= & \frac{1}{2} \int d \mathbf{r} d t \int d \mathbf{r}^{\prime} d t^{\prime} \delta n(\mathbf{r}, t) f_{\mathrm{XC}}\left(\mathbf{r}, t ; \mathbf{r}^{\prime}, t^{\prime}\right) \delta n\left(\mathbf{r}^{\prime}, t^{\prime}\right) \\
& +\frac{1}{4 !} \int d \mathbf{r} d t \int d \mathbf{r}^{\prime} d t^{\prime} \int d \mathbf{r}^{\prime \prime} \int d \mathbf{r}^{\prime \prime \prime} \delta n(\mathbf{r}, t) \delta n\left(\mathbf{r}^{\prime}, t\right) \\
& \times f_{\mathrm{XC}}^{\prime \prime}\left(\mathbf{r}, t ; \mathbf{r}^{\prime}, t ; \mathbf{r}^{\prime \prime}, t^{\prime} ; \mathbf{r}^{\prime \prime \prime}, t^{\prime}\right) \delta n\left(\mathbf{r}^{\prime \prime}, t^{\prime}\right) \delta n\left(\mathbf{r}^{\prime \prime \prime}, t^{\prime}\right) .
\end{aligned}
$$

Indeed, in this case one can express the fluctuation of the electron charge density in terms of the polarizations $\delta n(\mathbf{r}, t) \simeq$ $\sum_{l, \mathbf{k}} P_{l \mathbf{k}}(t) \varphi_{l \mathbf{k}}(\mathbf{r})$ and substitute it into Eq. (19), which results in Eq. (15). In derivation of Eq. (15) we took into account only the terms proportional to $P_{1}^{4}, P_{1}^{2} P_{2}^{2}$, and $P_{2}^{4}$ in the expression for the nonequilibrium exciton thermodynamic potential, because these terms give the largest contribution to the energy due to the minimal number of oscillating factors $\exp \left(i \omega_{l \mathbf{k}} t\right)$ in front of each $P_{l \mathbf{k}}(t)\left(\omega_{l \mathbf{k}}\right.$ is the corresponding exciton frequency), in analogy to the case of the two-component $\phi^{4}$ model [18]. We assume that the time arguments in the first two and the last two densities in the last term in $\delta E_{\mathrm{XC}}$ are equal. This approximation corresponds to the mapping of the effective action on the corresponding effective action of free biexcitons:

$\int d \mathbf{r} d t \int d \mathbf{r}^{\prime} d t^{\prime} B^{*}\left(\mathbf{r}, \mathbf{r}^{\prime}, t\right) D^{-1}\left(\mathbf{r}, \mathbf{r}^{\prime}, t ; \mathbf{r}^{\prime \prime}, \mathbf{r}^{\prime \prime \prime}, t^{\prime}\right) B\left(\mathbf{r}^{\prime \prime}, \mathbf{r}^{\prime \prime \prime}, t\right)$,

assuming that $B\left(\mathbf{r}, \mathbf{r}^{\prime}, t\right) \sim \delta n(\mathbf{r}, t) \delta n\left(\mathbf{r}^{\prime}, t\right)$ and that the inverse biexciton propagator $D^{-1}\left(\mathbf{r}, \mathbf{r}^{\prime}, t ; \mathbf{r}^{\prime \prime}, \mathbf{r}^{\prime \prime \prime}, t^{\prime}\right) \sim$ $f_{\mathrm{XC}}^{\prime \prime}\left(\mathbf{r}, t ; \mathbf{r}^{\prime}, t ; \mathbf{r}^{\prime \prime}, t^{\prime} ; \mathbf{r}^{\prime \prime \prime}, t^{\prime}\right)$.

\section{THE 2DFS AND A POSSIBILITY OF THE BIEXCITONIC STATES}

In order to find the 2DFS of the system in the case of a three-pulse excitation, one needs to find the third-order polarization $P_{l \mathbf{k}}^{(3)}$ given on the left-hand side of Eq. (15). This can be done by inserting the solution for the linear polarizations into the right-hand side of the equation and then by making the Fourier transform with respect to the delay $\tau$ and the measurement $t$ times, which results in the spectrum that depends on the frequencies $\omega$ and $\Omega$, correspondingly. For simplicity, we will assume that the initial pulse takes place at time $t_{1}=0$, while two other pulses occur at times $\tau>0$. The first-order polarization can be found by solving the equation which consists of the first two lines in Eq. (15). The solution is

$$
P_{l k}(t)=\int_{-\infty}^{t} e^{-i\left(\omega_{l k}-i \Gamma_{l k}\right)\left(t-t^{\prime}\right)} d_{k}^{c v_{1}} E\left(t^{\prime}\right) d t^{\prime},
$$

where $\omega_{l k}$ and $\omega_{l k}$ are the energy and the lifetime of the $l$ exciton.

In order to obtain biexcitons, one needs to consider a retarded exciton interaction $F\left(t-t^{\prime}\right)$ [6]. Before presenting the numerical results of the application of our approach, it is useful to discuss the conditions which $F\left(t-t^{\prime}\right)$ has to satisfy in order to obtain the biexcitonic peaks. As it was argued in Ref. [6], for this purpose in the similar equation for the polarization in the many-body case, the memory function can be approximated by

$$
F(t)=\int_{0}^{\infty} d \omega \rho(\omega) e^{-i \omega t}
$$

where "the heat bath" spectral density $\rho(\omega)$ in the lowfrequency limit, which defines the long-time asymptotic behavior of the system, can be approximated by a power function $\rho(\omega) \sim \omega^{\alpha}$. This function defines the dissipation processes for given exciton due to the environment consisting of the surrounding excitons. In the case when $\alpha$ is smaller, equal or larger than 1, the dissipation is called "sub-ohmic," "ohmic," and "super-ohmic," respectively [19]. Since the spectral function must decay at large frequencies, the general form of the spectral density was approximated by

$$
\rho(\omega)=A \omega^{\alpha} e^{-\omega / \omega_{F}},
$$

where $\omega_{F}$ is the frequency scale and $A$ is the normalization constant. In the case of a one-dimensional model for the excitons, the authors of Ref. [6] found $\alpha=1$.

In TDDFT the memory effects are described by the matrix elements of the first and second derivatives of the XC kernel. One can in principle construct a $f_{\mathrm{XC}}^{\prime \prime}$ to reproduce (22). In this case, the equation for the polarization and its solution, including the biexcitonic features, will coincide with the many-body case. While there are known constraints on the frequency-dependent (nonadiabatic) part of $f_{\mathrm{XC}}$ at small and large frequencies, the full dependence of $f_{\mathrm{XC}}^{\prime \prime}$ has not been discussed in detail so far. In particular, it is known that the exact asymptotic of the $\mathrm{XC}$ kernel at large frequencies is $f_{\mathrm{XC}} \sim a+b \omega^{-2}$ [20]. In the low-frequency limit, the information about the exact behavior of $f_{\mathrm{XC}}$ is more limited. It is known that it can have poles in the discrete part of the spectrum in the case of a finite system. One can construct the frequency dependence of the XC kernel for all ranges of frequencies by using results of the homogeneous electron gas: $f_{\mathrm{XC}}(\omega \rightarrow 0) \rightarrow 0, f_{\mathrm{XC}}(\omega \rightarrow \infty) \rightarrow \omega^{-3 / 2}$ [21]. From these results one can suggest the following rather general form for 
the nonadiabatic part of the XC kernel:

$$
f_{\mathrm{XC}}(\omega)=A \frac{\omega^{\alpha}}{1+\left(\omega / \omega_{F}\right)^{\alpha+\beta}},
$$

where $\alpha>0$ and $\beta=2$, though $\beta=3 / 2$ is also worth special attention. Since our main goal is to explore the role of nonadiabaticity in the nonlinear response, we assume that full kernel is the product of the frequency part Eq. (24) and the spatial part $\tilde{f}\left(\mathbf{r}, \mathbf{r}^{\prime}\right): f_{\mathrm{XC}}(\mathbf{r}, \omega)=A\left\{\omega^{\alpha} /\left[1+\left(\omega / \omega_{F}\right)^{\alpha+\beta}\right]\right\} \tilde{f}\left(\mathbf{r}, \mathbf{r}^{\prime}\right)$, which allows us to separate the spatial and temporal contributions to the interaction, and which makes the analysis more transparent. A more general case of the kernel may be considered, though we believe that main nonadiabatical effects in the many-exciton system can be captured by this function.

It is also natural to assume that the retardation effects in the exciton case $\left(f_{\mathrm{XC}}^{\prime \prime}\right)$ are described by the same time dependence as in the electron case $\left(f_{\mathrm{XC}}\right)$, because the excitons are composed of "the elementary" quasiparticles, electrons and holes, interacting through the retarded interaction given above. Indeed, using the frequency-dependence Eq. (24) for $f_{\mathrm{XC}}^{\prime \prime}$ one can demonstrate that the corresponding 2DFS includes biexcitonic features, similar to the many-body approximation case [Eqs. (22) and (23)] [6]. However, using such an approximation gives rise to many questions about the physical meaning of the corresponding spectral function, in particular: Can one assume that the heat- (or exciton-) bath dissipation results in the formation of biexcitonic states? This question is nontrivial and deserve a deep study. In this paper we use a simplified form of $F(t)$ by making the analogy with the many-body theory, where the biexciton propagator $D$ in Eq. (20) must contain poles at the biexciton frequencies. This means we postulate that such poles must be present in $f_{\mathrm{XC}}^{\prime \prime}$. Namely, we approximate

$$
\begin{aligned}
& f_{\mathrm{XC}}^{\prime \prime}\left(\mathbf{r}, t, \mathbf{r}^{\prime}, t^{\prime}, \mathbf{r}^{\prime \prime}, t^{\prime \prime}, \mathbf{r}^{\prime \prime \prime}, t^{\prime \prime \prime}\right) \\
& \quad \simeq g_{\mathrm{XC}}\left(t-t^{\prime \prime}\right) \delta\left(t-t^{\prime}\right) \delta\left(t^{\prime \prime}-t^{\prime \prime \prime}\right) \tilde{g}\left(\mathbf{r}, \mathbf{r}^{\prime}, \mathbf{r}^{\prime \prime}, \mathbf{r}^{\prime \prime \prime}\right) .
\end{aligned}
$$

The approximation in the time dependence of the kernel derivative corresponds to taking into account only the two-particle interactions in the XC energy (19). Namely, it is assumed that "the quasiparticle" $\delta n(\mathbf{r}, t) \delta n\left(\mathbf{r}^{\prime}, t\right)$ at time $t$ (two electrons at points $\left.\mathbf{r}, \mathbf{r}^{\prime}\right)$ interacts with the one $\delta n\left(\mathbf{r}^{\prime \prime}, t^{\prime \prime}\right) \delta n\left(\mathbf{r}^{\prime \prime \prime}, t^{\prime \prime}\right)$ at time $t^{\prime \prime}$ (two holes at points $\mathbf{r}^{\prime \prime}, \mathbf{r}^{\prime \prime}$ ), which can be easily seen from Eq. (19):

$$
\begin{aligned}
E_{\mathrm{XC}} \sim & \frac{1}{4 !} \int d \mathbf{r} d t \int d \mathbf{r}^{\prime} d t^{\prime} \int d \mathbf{r}^{\prime \prime} \int d \mathbf{r}^{\prime \prime \prime} \delta n(\mathbf{r}, t) \delta n\left(\mathbf{r}^{\prime}, t\right) \\
& \times g_{\mathrm{XC}}\left(t-t^{\prime \prime}\right) \tilde{g}\left(\mathbf{r}, \mathbf{r}^{\prime}, \mathbf{r}^{\prime \prime}, \mathbf{r}^{\prime \prime \prime}\right) \delta n\left(\mathbf{r}^{\prime \prime}, t^{\prime \prime}\right) \delta n\left(\mathbf{r}^{\prime \prime \prime}, t^{\prime \prime}\right) .
\end{aligned}
$$

In this case, the pole structure of $f_{\mathrm{XC}}^{\prime \prime}(\omega)$ is contained in $g_{\mathrm{XC}}(\omega)$ :

$$
g_{\mathrm{XC}}(\omega)=\sum_{l, k, m, q} \frac{A_{l, k, m, q}}{\omega-\omega_{B l, k, m, q}+i \Gamma_{B l, k, m, q}},
$$

where $A_{l, k, m, q}$ is the weight, and $\omega_{B l, k, m, q}, \Gamma_{B l, k, m, q}$ are the spectrum and the decay rate for the biexciton state formed by two excitons described by the orbital $l, m$ and the momentum $\mathbf{k}, \mathbf{q}$ quantum numbers. Indeed, the pole structure (27) guarantees that the largest contribution to $\delta E_{\mathrm{XC}}$ [Eq. (26)] comes from the states at the resonant frequencies, e.g., the two excited-electron, two-exciton, and in our case, the biexciton frequencies. This is a physically reasonable situation, since we are assuming that there are only exciton or two-exciton (biexcitonic) excitations in the system. The imaginary part of the structure (27) may be regarded as a particular case of the Lorentzian type kernel (24).

A pole structure of $f_{\mathrm{XC}}$ capable of describing the multiple excitations with TDDFT was considered, for example, in Ref. [16]. In this paper we propose a similar structure for the kernel second derivative $f_{\mathrm{XC}}^{\prime \prime}$ [Eq. (27)]. In Eq. (27) we again separate the spatial and temporal parts for the sake of simplicity of the analysis. From Eq. (27) one can easily obtain the structure of the corresponding nonadiabatic part of the $\mathrm{XC}$ kernel:

$$
\begin{aligned}
f_{\mathrm{XC}}\left(\mathbf{r}, t, \mathbf{r}^{\prime}, t^{\prime}\right) \simeq & \frac{1}{4 !} \int d \mathbf{r} d t \int d \mathbf{r}^{\prime} \int d \mathbf{r}^{\prime \prime} d t^{\prime \prime} \int d \mathbf{r}^{\prime \prime \prime} \\
& \times \delta n(\mathbf{r}, t) \delta n\left(\mathbf{r}^{\prime}, t\right) g_{\mathrm{XC}}\left(t-t^{\prime \prime}\right) \tilde{g}\left(\mathbf{r}, \mathbf{r}^{\prime}, \mathbf{r}^{\prime \prime}, \mathbf{r}^{\prime \prime \prime}\right) \\
& \times \delta n\left(\mathbf{r}^{\prime \prime}, t^{\prime \prime}\right) \delta n\left(\mathbf{r}^{\prime \prime \prime}, t^{\prime \prime}\right) .
\end{aligned}
$$

\section{SOLUTION}

We apply the formalism above to analyze the effect of the memory function on the 2DFS of a GaAs multiple quantum well and compare the results with experimental data [22]. The result for the spectrum depends on the heavy and light exciton frequencies and lifetimes $\omega_{\mu}$ and $\Gamma_{\mu}(\mu=$ $h, l$ ), and on the memory function $F(t)$. DFT calculations with the Quantum Espresso code [23] with LDA-PZ81 XC potential [24], BHS norm-conserving pseudopotential and $11 \times 11 \times 11$ set of $\mathrm{k}$-points were used to generate the static wave functions [since the width of the quantum well is usually rather large $(>10 \mathrm{~nm})$, we used the bulk approximation to generate the wave functions]. With this input we used also the

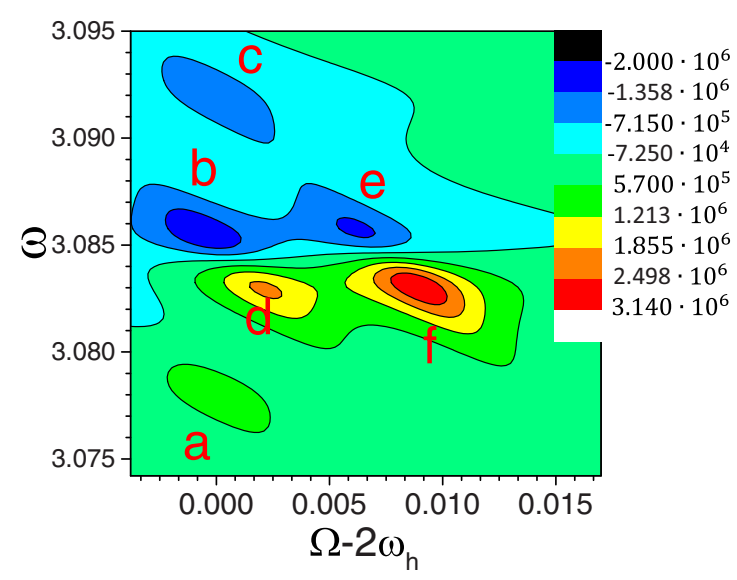

FIG. 1. (Color online) The 2DFS (the imaginary part) of the GaAs multiwell system. The frequencies $\omega$ and $\Omega$ are given in meV. The features in the spectrum are marked as follows: (a) two heavy excitons, (b) a mixed (heavy + light) exciton, (c) two light excitons, (f) a mixed heavy-light biexciton. The features (d) and (e) correspond to an exciton-biexciton coherence. The spectrum was obtained by using the kernel (27) with the weights $A$ defined by static values (17) and (18) and the pole at the frequency $\omega_{B l, k, m, q}=$ $\omega_{h}+\omega_{l}-E_{X X}$, with the parameters given in the text (we neglected the momentum dependence of the excitations). 
experimental results for the heavy and light exciton frequencies $\omega_{h}=1.539 \mathrm{eV}, \omega_{l}=1.546 \mathrm{eV}$ and for the heavy-light hole biexciton binding energy $E_{X X}=1.5 \mathrm{meV}$ [22]. We use one value $\Gamma=1 \mathrm{meV}$ for the exciton and biexciton lifetimes, which agrees with the experimental estimations [2]. Finally, we use $f_{\mathrm{XC}}^{\prime \prime}$ defined in Eq. (25) with the memory function (27), and for the spatial part we use the adiabatic LDA approximation:

$$
\begin{aligned}
\tilde{g}\left(\mathbf{r}, \mathbf{r}^{\prime}, \mathbf{r}^{\prime \prime}, \mathbf{r}^{\prime \prime \prime}\right) & =\delta^{2} f_{X}^{\mathrm{LDA}}\left(\mathbf{r}, \mathbf{r}^{\prime}\right) / \delta n\left(\mathbf{r}^{\prime \prime}\right) \delta n\left(\mathbf{r}^{\prime \prime \prime}\right) \\
& =f_{X}^{\mathrm{LDA}^{\prime \prime}}(\mathbf{r}) \delta\left(\mathbf{r}-\mathbf{r}^{\prime}\right) \delta\left(\mathbf{r}-\mathbf{r}^{\prime \prime}\right) \delta\left(\mathbf{r}-\mathbf{r}^{\prime \prime \prime}\right) .
\end{aligned}
$$

We compare the solutions with both adiabatic and nonadiabatic $g_{\mathrm{XC}}$ 's. As the main result, it follows from our calculations that it is impossible to obtain the nondiagonal (biexciton) peaks using an instant (adiabatic) exciton-exciton interaction. In contrast, it is absolutely necessary to use the nonadiabatic exciton-exciton interaction in order to obtain two diagonal states (that correspond to two heavy and two light excitonic peaks) and the nondiagonal (heavy-light exciton, as in cocircular excitation experiment [22]) bound state in the 2DFS (Fig. 1). Our results shown in Fig. 1 are in agreement with the experimental data [22].

\section{CONCLUSIONS}

We have derived a TDDFT version of the nonlinear equation for the exciton dynamics. We have found that the effective time-dependent exciton-exciton interaction is defined by the nonadiabatic part of the $\mathrm{XC}$ kernel and discussed possible time dependencies of this interaction. We applied the approach to study the 2DFS of a GaAs quantum well system and showed that the main features of the spectrum, including biexcitons, can be reproduced within a TDDFT approach. The densitymatrix version of TDDFT developed in the paper can be used to analyze the nonlinear ultrafast response of different types of many-fermion systems. The technical simplicity and physical transparency of this ab initio approach makes it favorable compared to the currently used many-body techniques. In most cases, the nonadiabatic (memory) effects play a crucial role in the ultrafast response. The proposed theory, when applied to analyze the 2DFS and other ultrafast experiments, may lead to an improvement of our understanding of the structure of the nonadiabatic XC kernel for different types of systems where these effects play an important role.

\section{ACKNOWLEDGMENTS}

We would like to thank Dr. Mikhail Erementchouk for numerous enlightening discussions. This work was supported in part by Grants No. NSF-ECCS 072551, No. NSF-ECCS0901784, No. NSF-ECCS-1128597, AFOSR No. FA9550-091-0450, DARPA/MTO Young Faculty Award HR0011-08-10059 (M.N.L.), and No. DOE-DE-FG02-07ER15842 (V.T.).
[1] G. Onida, L. Reining, and A. Rubio, Rev. Mod. Phys. 74, 601 (2002); F. Rossi and T. Kuhn, ibid. 74, 895 (2002).

[2] M. Erementchouk, M. N. Leuenberger, and L. J. Sham, Phys. Rev. B 76, 115307 (2007); 79, 085307 (2009).

[3] V. Z. Tronciu and R. A. Abram, Phys. Rev. E 65, 026616 (2002).

[4] X. Li, Ya. Wu, D. Steel et al., Science 301, 809 (2003).

[5] Th. Ostreich, K. Schonhammer, and L. J. Sham, Phys. Rev. Lett. 74, 4698 (1995); Phys. Rev. B 58, 12920 (1998).

[6] Th. Ostreich and L. J. Sham, Phys. Rev. Lett. 83, 3510 (1999).

[7] E. Runge and E. K. U. Gross, Phys. Rev. Lett. 52, 997 (1984).

[8] Y. H. Kim and A. Görling, Phys. Rev. Lett. 89, 096402 (2002); Phys. Rev. B 66, 035114 (2002).

[9] H. Haug and S. W. Koch, Quantum Theory of the Optical and Electronic Properties of Semiconductors (World Scientific, Singapore, 2001).

[10] V. Turkowski and C. A. Ullrich, Phys. Rev. B 77, 075204 (2008).

[11] V. Turkowski, A. Leonardo, and C. A. Ullrich, Phys. Rev. B 79, 233201 (2009).

[12] V. Turkowski, C. A. Ullrich, T. S. Rahman, and M. N. Leuenberger, Phys. Rev. B 82, 205208 (2010).
[13] K. Pernal, O. Gritsenko, and E. J. Baerends, Phys. Rev. A 75, 012506 (2007).

[14] K. J. H. Giesbertz, E. J. Baerends, and O. V. Gritsenko, Phys. Rev. Lett. 101, 033004 (2008).

[15] K. J. H. Giesbertz, K. Pernal, O. V. Gritsenko, and E. J. Baerends, J. Chem. Phys. 130, 114104 (2009).

[16] N. T. Maitra, F. Zhang, R. J. Cave, and K. Burke, J. Chem. Phys. 120, 5932 (2004).

[17] Y. Li and C. A. Ullrich, Chem. Phys. 391, 157 (2011).

[18] V. M. Turkowski, P. D. Sacramento, and V. R. Vieira, Phys. Rev. B 73, 214437 (2006).

[19] A. O. Caldeira and A. J. Leggett, Physica A 121, 587 (1983).

[20] R. van Leeuwen, Int. J. Mod. Phys. B 15, 1969 (2001).

[21] M. A. L. Marques and E. K. U. Gross, in A Primer in DensityFunctional Theory, edited by C. Fiolhais, F. Nogueira, and M. A. L. Marques, Lecture Notes in Physics, Vol. 620 (Springer, Berlin, 2003), p. 144.

[22] K. W. Stone, K. Gundoglu, D. B. Turner et al., Science 324, 1169 (2009).

[23] P. Giannozzi et al., J. Phys.: Condens. Matter 21, 395502 (2009).

[24] J. P. Perdew and A. Zunger, Phys. Rev. B 23, 5048 (1981). 